\title{
Survivin nuclear labeling index: a superior biomarker in superficial urothelial carcinoma of human urinary bladder
}

\author{
Wu Yin ${ }^{1,4}$, Ni Chen ${ }^{1,2,4}$, Yutao Zhang ${ }^{1,4}$, Hao Zeng ${ }^{2,3}$, Xueqin Chen ${ }^{1}$, Yongtao He ${ }^{1,2}$, \\ Xiaojie Wang ${ }^{1}$ and Qiao Zhou ${ }^{1,2}$ \\ ${ }^{1}$ Department of Pathology, West China Hospital, West China Medical School, Sichuan University, Chengdu, \\ China; ${ }^{2}$ Laboratory of Pathology, National Key Laboratory, West China Hospital, Sichuan University, \\ Chengdu, China and ${ }^{3}$ Department of Urology, West China Hospital, West China Medical School, \\ Sichuan University, Chengdu, China
}

\begin{abstract}
The caspase family proteases are key proapoptotic proteins while the inhibitor of apoptosis proteins (IAP) prevent apoptosis by antagonizing the caspases or other key proapoptotic proteins. Limited studies of IAPs suggested their deregulation contributed to urothelial neoplasia. However, the expression status and biologic or prognostic significance of the caspase and IAP family proteins in urothelial neoplasms is not clear. In the present study, we first systematically evaluated the expression profile of the major apoptosis regulators, including caspases (CASP3, 6, 7, 8, 9, 10, and 14), IAPs (survivin/BIRC5, CIAP1, CIAP2, XIAP, and LIVIN), APAF1, SMAC, and BCL2, as well as proliferation markers Ki67 and PHH3, in Ta/T1 human urinary bladder urothelial carcinomas and normal urothelium samples by immunohistochemistry. The analysis showed that survivin/ BIRC5 nuclear labeling index (BIRC5-N), but not cytoplasmic staining, was the only apoptotic marker which correlated significantly with tumor grade, stage, and patient outcome. We further analyzed the prognostic value of BIRC5-N in $101 \mathrm{Ta} / \mathrm{T} 1$ urinary bladder urothelial carcinomas by univariate analysis, which showed that BIRC5$\mathrm{N}$ as well as the more classical prognosticators (stage, grade, and Ki67 index) were of prognostic significance. However, multivariate analysis by Cox proportional hazard regression demonstrated BIRC5-N was a stronger prognosticator than tumor grade, stage, and Ki67 labeling index. BIRC5-N index of $8 \%$ or more predicted unfavorable disease-specific survival (relative risk $(\mathrm{RR})=6.6,95 \%$ confidence interval $=1.6-26.7, P=0.0080$ ) as well as progression-free survival $(R R=4.4,95 \%$ confidence interval $=1.3-14.6, P=0.0151)$. We conclude that BIRC5- $\mathrm{N}$ is a superior biologic and prognostic marker for $\mathrm{Ta} / \mathrm{T} 1$ urothelial carcinomas of urinary bladder. Modern Pathology (2006) 19, 1487-1497. doi:10.1038/modpathol.3800675; published online 4 August 2006
\end{abstract}

Keywords: survivin/BIRC5; urothelial carcinoma; urinary bladder; prognosis

Urothelial carcinoma is the most common cancer of human urinary bladder and is among the major malignancies. ${ }^{1}$ Many efforts have been made in search of biomarkers associated with histopathological features and biological behavior of urothelial carcinomas, ${ }^{2,3}$ in order to achieve accurate grading, staging, and optimal management. Molecules involved in cell death and proliferation are among

Correspondence: Dr Q Zhou, MD, PhD, Department of Pathology, West China Hospital, West China Medical School, Sichuan University, Chengdu, Sichuan 610041, China.

E-mail: zhouqiao@mcwcums.com

Preliminary results of the project was submitted in meeting abstract form to the United States and Canadian Academy of Pathology Annual Meeting, Atlanta, Georgia, February, 2006.

${ }^{4}$ These authors made equal contribution to this work.

Received 15 May 2006; revised 13 July 2006; accepted 18 July 2006; published online 4 August 2006 those receiving more attention, ${ }^{4-27}$ but their roles in urothelial carcinogenesis and tumor progression are not well understood.

The caspase family proteases are key proteins in apoptotic signaling pathways, ${ }^{28-30}$ while the intrinsic inhibitor of apoptosis proteins (IAP) effectively prevent apoptosis by antagonizing the caspases or inhibiting other proapoptotic proteins, in addition to participating in cell cycle progression and control of cell division. ${ }^{31,32}$ In addition, mitochondrial proteins APAF1 and SMAC are also key proapoptotic proteins released upon apoptotic stimuli, which are involved in caspase activation and inhibition of IAPs, respectively. ${ }^{33}$ Limited studies of a few of the IAP family proteins in urothelial carcinomas suggested their deregulation might contribute to urothelial neoplasia. ${ }^{22-24,26,27}$ Researchers are also exploring experimental approaches targeting 
Survivin/BIRC5 to induce bladder cancer cell death. $^{34}$ However, systematic evaluation of the expression profile and biologic significance of these apoptosis regulators in urothelial carcinomas is lacking. In addition, although tumor cell proliferation (as assessed by the immunomarker MIB1/Ki67) has been correlated with urothelial carcinoma progression and patient survival, $4,11,14,17,21,25$ the relationship of proliferation activity with caspase and IAP expression in urothelial carcinomas is not clear.

The present study aimed to evaluate comprehensively the expression profile of apoptosis regulators in Ta and T1 urothelial carcinoma of human urinary bladder, and to identify apoptotic regulators most closely associated with proliferation activity, degree of malignancy and patient outcome.

\section{Materials and methods}

\section{Tissue and Cell Samples}

Cases were from the authors' institution, and all tissues were collected and used strictly in accordance with institutional guidelines. Consecutive cases were evaluated for tissue adequacy, accuracy of staging, and therapeutic protocols. Fifty-nine low-grade (LGC) and 42 high-grade (HGC), pathologically confirmed Ta (54 cases) and T1 (47 cases) human urinary bladder urothelial carcinomas, and 11 normal urothelial samples were included. Carcinoma in situ was excluded. Diagnosis was pathologically established by complete transurethral resection of bladder tumor (TURBT). These patients received weekly intravesical instillation of bacillus Calmette-Guerin (BCG) for 6 consecutive weeks, starting 2 weeks after resection of tumor, without additional adjuvant therapies. Formalin-fixed, paraffin-embedded tissues were used for immunohistochemistry. Fresh frozen tissues from five paired urothelial carcinoma and tumor-free adjacent urothelium samples, and four additional normal urothelium samples from cystectomy performed for non-neoplastic conditions were snap frozen in liquid nitrogen and were used for RT-PCR and Western blot analyses. All cases were reviewed and graded according to the WHO/ISUP criteria. ${ }^{3}$ The urothelial carcinoma cell lines T24 (high-grade) and RT4 (low-grade) (from ATCC) were cultured in RPMI1640 and McCoy's 5A medium with 10\% fetal calf serum, respectively.

\section{Antibodies}

The following antibodies at indicated dilutions were used: CASP3 (rabbit polyclonal, Santa Cruz, Santa Cruz, CA, USA, 1:250), CASP6 (goat polyclonal, Santa Cruz, 1:100), CASP7 (goat polyclonal, Santa Cruz, 1:100), CASP8 (goat polyclonal, Santa Cruz, 1:100), CASP9 (rabbit polyclonal, Santa Cruz,
1:100), CASP10 (goat polyclonal, Santa Cruz, 1:100), CASP14 (goat polyclonal, Santa Cruz, 1: 300), CIAP1 (rabbit polyclonal, Santa Cruz, 1:100), CIAP2 (rabbit polyclonal, Santa Cruz, 1:100), XIAP (rabbit polyclonal, ProteinTech Group Inc., Chicago, IL, USA, 1:300), Survivin/BIRC5 (rabbit polyclonal, R\&D Systems Inc., Minneapolis, MN, USA, 1:300 for IHC, and 1:1000 for immunoblotting), Livin (goat polyclonal, R\&D, 1:500), APAF1 (rabbit polyclonal, Santa Cruz, 1:100), SMAC (goat polyclonal, Santa Cruz, 1:300), BCL2 (mouse monoclonal, Zymed Laboratories Inc., San Francisco, CA, USA, 1:200), PHH3 (rabbit polyclonal, Upstate Group LLC, Charlottesville, VA, USA, 1:500), Ki67(MIB1) (mouse monoclonal, DakoCytomation, Glostrup, Denmark, 1:100), GAPDH (mouse monoclonal, clone 6C5, Kangcheng, Shanghai, China, 1:10000).

\section{Immunohistochemistry}

Sections $(4 \mu \mathrm{m})$ were immunostained by standard labeled streptavidin-biotin protocol. Omission of primary antibodies was used as a control. A procedure to block endogenous avidin-binding activity $^{35}$ was employed. Antigen retrieval was by high-pressure boiling in citrate buffer ( $\mathrm{pH}$ 6.0) for 3 min. Immunostaining for each marker was performed in one batch with appropriate positive and negative controls. Reagents and reaction conditions were carefully controlled to minimize variations across batches. Cytoplasmic staining of the apoptotic markers showed a diffuse staining pattern when positive and was scored by conventional four-tiered semiquantitative scoring system (scores $0-3$ for negative, weak, moderate, and strong staining, respectively), ${ }^{36}$ based on staining intensity. Survivin/BIRC5 displayed distinct nuclear and cytoplasmic staining, and was separately scored as BIRC5-C and BIRC5-N. The staining of proliferative markers Ki67 and PHH3 was exclusively nuclear. The BIRC5 nuclear labeling index (BIRC5-N, \%) and Ki67 labeling index (\%) was determined by counting positive cells in 1000 cells, starting from the most intensely labeled region. PHH3 positivity (positive cells/10 high-power fields) was determined by counting labeled cells in at least 10 (and up to 50) high-power fields also starting from the most intensely labeled region. Immunostaining was evaluated and scored by two pathologists independently and then cross-checked.

\section{Reverse Transcriptase-Polymerase Chain Reaction}

Primers for caspases and IAPs were designed according to cDNA sequences in GenBank and synthesized by Invitrogen (Shanghai, China). Primers (upstream and downstream) for the target genes were: CASP3 (5'-CTGGTACAGATGTCGATGC AGC-3' ${ }^{\prime}$ 5'-AAGAAGTCGGCCTCCACTG-3'), CASP6 (5'-TCAGACAGAGAAGTTGGACACC- ${ }^{\prime}, 5^{\prime}$-CTGTG 
AACTCTAAGGAGGAGCC-3'), CASP8 (5'-GCCTGC TGAAGATAATCAACG-3' ${ }^{\prime} 5^{\prime}$-CTTTCTCCCGTGCTT TTGC-3'), CASP9 (5'-CCACACCCAGTGACATCTT TG-3', 5'-ACCGAAACAGCATTAGCGAC-3'), CIAP1 (5'-TTGTCAACTTCAGATACCACTGGAG-3', 5'-CA AGGCAGATTTAACCACAGGTG-3'), CIAP2 (5'-AGG GAAGAGGAGAGAGAAAGAGC-3', 5'-CGGCAGTT AGTAGACTATCCAGG-3'), Survivin/BIRC5 (5'-GC

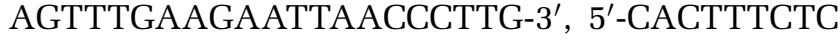
CGCAGTTTCCTC- $\left.3^{\prime}\right)$, XIAP (5'-GGGTTCAGTTTC AAGGACATTAAG-3', 5'-CGCCTTAGCTGCTCTTCA GTAC- $3^{\prime}$ ). $\beta$-Actin was used as internal control (5'TGGAGAAAATCTGGCACCAC-3', 5 '-GAGGCGTA CAGGGATAGCAC-3'). Primers were designed to span introns to avoid false positivity from genomic DNA contamination. Positive controls using cultured cancer cell lines known to express these genes and negative controls by omission of templates were employed. Total RNA from fresh tissue and cultured cells was isolated with the Trizol reagent (Invitrogen, Carlsbad, CA, USA). Reverse transcription was carried out in $20 \mu \mathrm{l}$ mixture containing $5 \mu \mathrm{g}$ total RNA, $0.5 \mu \mathrm{g}$ oligo(dT) $)_{18}$ primer, $2 \mu \mathrm{l}$ of $10 \mathrm{mmol} / \mathrm{l}$ dNTP, $0.1 \mu \mathrm{l}$ of $1 \mathrm{~mol} / \mathrm{l}$ DTT, and $1 \mu \mathrm{l}$ of M-Mulv reverse transcriptase (Fermentas, Hanover, MD, USA), for $60 \mathrm{~min}$ at $42^{\circ} \mathrm{C}$ followed by $10 \mathrm{~min}$ at $72^{\circ} \mathrm{C}$. PCR was carried out with Taq DNA polymerase (Takara, Japan) in 30 cycles of amplification (30 s at $95^{\circ} \mathrm{C}, 30 \mathrm{~s}$ at predetermined appropriate annealing temperature for each primer set, and $35 \mathrm{~s}$ at $72^{\circ} \mathrm{C}$ ), followed by $10 \mathrm{~min}$ at $72^{\circ} \mathrm{C}$. PCR products were resolved by $2 \%$ agarose gel and visualized by ethidium bromide staining.

\section{Western Blot Analysis}

Fresh frozen tissue samples were minced and powdered with mortar and pestle on liquid nitrogen. Total proteins from powdered tissue samples and cultured cells were extracted in the presence of protease inhibitor cocktails (Roche Diagnostics, Mannheim, Germany), quantitated by using the BCA kit (Pierce Biotechnology Inc., Rockford, IL, USA) and resolved by $10 \%$ SDS polyacrylamide (Sigma, St Louis, MO, USA) gel electrophoresis. Proteins were electroblotted to PVDF membrane (Amersham Biosciences UK Ltd., Little Chalfont, UK) in CAPS buffer (pH 11.0) (Amresco, Solon, OH, USA), and then incubated with block solution ( $5 \%$ non-fat milk, $0.1 \%$ Tween 20 , in $1 \times$ TBS, Sigma, St Louis, MO, USA) at room temperature for $2 \mathrm{~h}$. Anti-BIRC5/survivin was used at 1:1000, antiGAPDH (mouse monoclonal, clone 6C5, Kangcheng, Shanghai, China, 1:10000) was used as internal control. Horseradish peroxidase-labeled secondary antibodies were from Zymed (San Francisco, CA, USA). Incubation with primary and secondary antibodies were at room temperature for 2 and $1.5 \mathrm{~h}$, respectively. Signals were detected by exposure to X-ray films after treatment with the SuperSignal enhanced chemiluminescence kit (Pierce Biotechnology Inc., Rockford, IL, USA) after incubation with primary and secondary antibodies.

\section{Statistical Analysis}

General statistical and survival analysis was carried out by using the Statistica software (StatSoft Inc., Tulsa, OK, USA). Intergroup differences were examined by using Student's $t$-test or Mann-Whitney $U$-test, as appropriate. Spearman rank order correlation was used to examine correlations between variables.

\section{Results}

\section{Overview of Expression Profile of Apoptosis Regulators and Proliferation Markers in Ta/T1 Urothelial Carcinomas}

Our first analysis consisted of an overview of the immunoprofiles of apoptosis regulators and proliferation markers (together with mitotic index) in $37 \mathrm{Ta} / \mathrm{T} 1$ urinary bladder urothelial carcinomas and 11 normal urothelium samples (Figure 1), RT-PCR analysis of mRNA of selected apoptosis regulators and Western blot analysis of survivin/BIRC5 protein in fresh tissue samples (Figure 2).

Representative immunostaining was shown in Figure 1a. Most of the apoptosis regulators showed a cytoplasmic staining pattern, with the exception of survivin/BIRC5, which displayed distinct cytoplasmic (BIRC5-C) and nuclear (BIRC5-N) staining patterns (Figure 1b). The immunostaining results of these markers were visually summarized in a color scale map (Figure 3a).

In parallel with the immunohistochemical analysis, we examined the mRNA expression by RT-PCR of CASP3, 6, 8, and 9, and CIAP1, CIAP2, BIRC5, and XIAP, in 10 paired fresh specimens of highgrade urothelial carcinoma and adjacent normal urothelium together with the urothelial carcinoma cell-line T24 (Figure 2, upper panel). Semiquantitative analysis showed that mRNA of these IAPs and caspases, particularly BIRC5 and CASP3, was more abundant in urothelial carcinomas than in normal urothelium. Western blot analysis (Figure 2, lower panel) demonstrated abundant Survivin/BIRC5 protein in fresh tumor samples and urothelial carcinoma cell lines T24 and RT4, but not in normal urothelium samples.

Comparison of the immunostaining data (Figure 3a) between normal urothelium and LGC or HGC was summarized in Figure $3 \mathrm{~b}$. The urothelial carcinomas had much higher levels of antiapoptotic proteins BIRC5 (both BIRC5-C and BIRC5-N), CIAP1, and CIAP2, as well as the proapoptotic protein CASP3 (all with $P<0.01$ ) than normal urothelium. Expression of other apoptosis regulatory proteins were not significantly different between carcinomas and normal urothelium. 

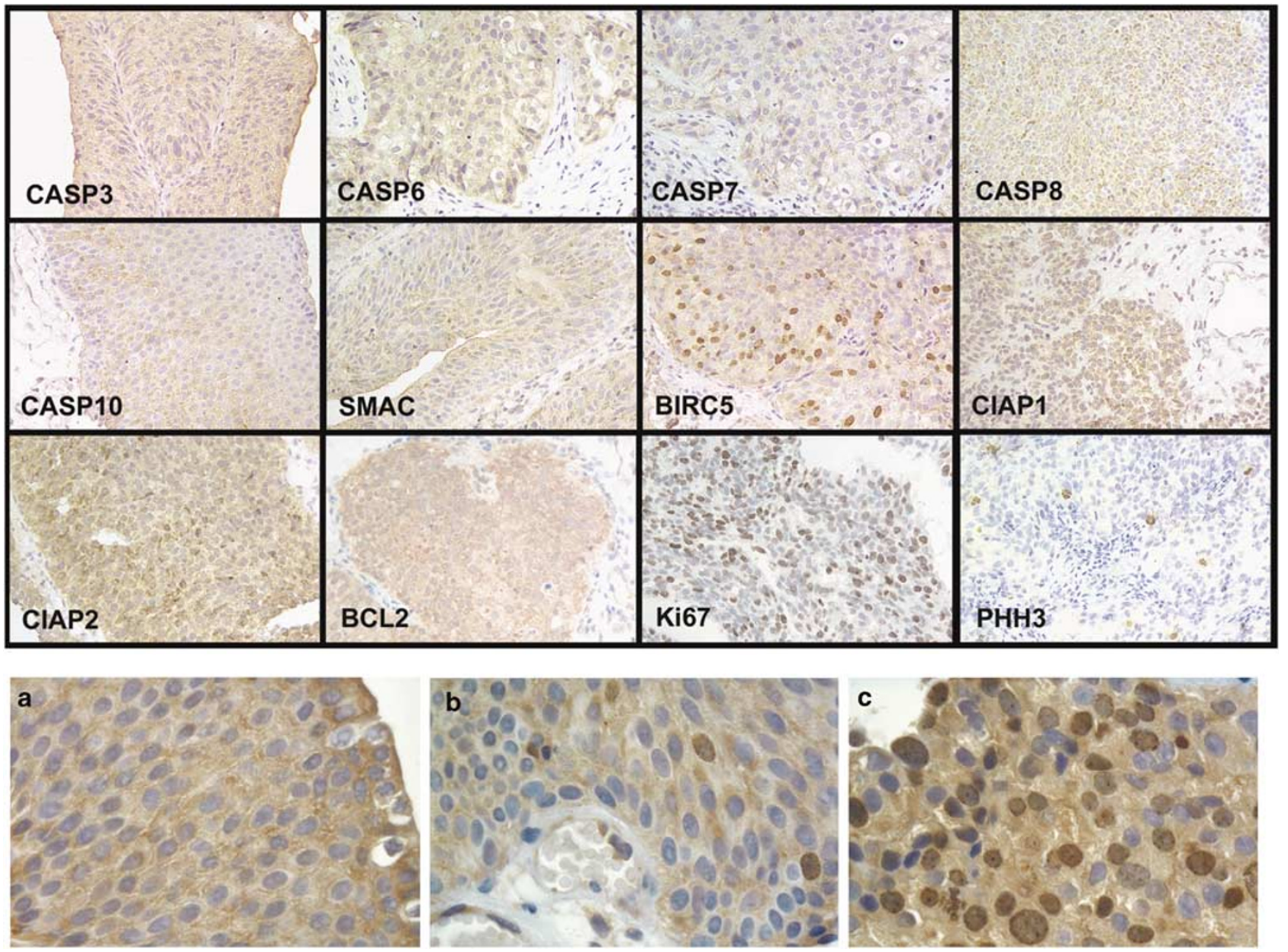

Figure 1 Upper panel. Representative positive immunostaining of apoptosis regulators and proliferation markers in Ta/T1 urothelial carcinomas. Horseradish peroxidase labeled streptavidin-biotin immunostaining with hematoxylin counterstaining. Notice the distinct nuclear and cytoplasmic staining of Survivin/BIRC5. Original magnification for each panel: $\times 400$. Lower panel. Higher power view of BIRC5 staining patterns in urothelial carcinoma. (a) Only cytoplasmic staining was observed in this case. (b) This case has low BIRC5-N index. Occasional nuclear labeling in addition to cytoplasmic staining. Notice also typical staining in vascular endothelial cells. (c) A tumor with high BIRC5-N index. Numerous nuclei were BIRC5 positive. Original magnification for each figure in lower panel: $\times 1000$.

Pairwise correlation analysis showed that the proliferation markers (Ki67, PHH3, and MF) were significantly associated with BIRC5-N expression in the carcinomas $\left(r_{\mathrm{s}}=0.7565,0.6692,0.6280\right.$, respectively, $P<0.01$ ), but not with BIRC5-C, nor with other apoptosis regulators. In addition, BIRC5-N was not significantly associated with the other IAPs or the caspases.

\section{Comparison between LGC and HGC}

When LGC and HGC were compared (Figure 3b), only BIRC5-N (but not BIRC5-C) and the proliferation markers were significantly different between the two grades $(P<0.01)$, but other markers were not.

\section{Comparison between Ta and T1}

Figure 3c summarized comparison of these markers between Ta and T1 carcinomas. Again, only BIRC5-N (but not BIRC5-C) and the prolifera- tion markers were significantly different between Ta and T1 carcinomas $(P<0.01)$, but other markers were not.

\section{Relationship of BIRC5-N and BIRC5-C Expression with Clinicopathological Parameters and Proliferation Activity}

The above initial analyses showed that BIRC5-N and proliferation markers were the prominent parameters associated with grade and stage of $\mathrm{Ta} / \mathrm{T} 1$ urinary bladder urothelial carcinomas, as well as prognosis (not shown). We then focused further analysis on the relationship of BIRC5 with tumor grade, stage, and proliferative activity, in a cohort of $101 \mathrm{Ta} / \mathrm{T} 1$ urinary bladder urothelial carcinomas. Table 1 summarized the relationship of BIRC5-N and BIRC5-C with clinicopathological parameters, which validated the initial result that BIRC5-N, but not BIRC5-C, was significantly associated with grade, stage, and proliferative activity. Notably, 


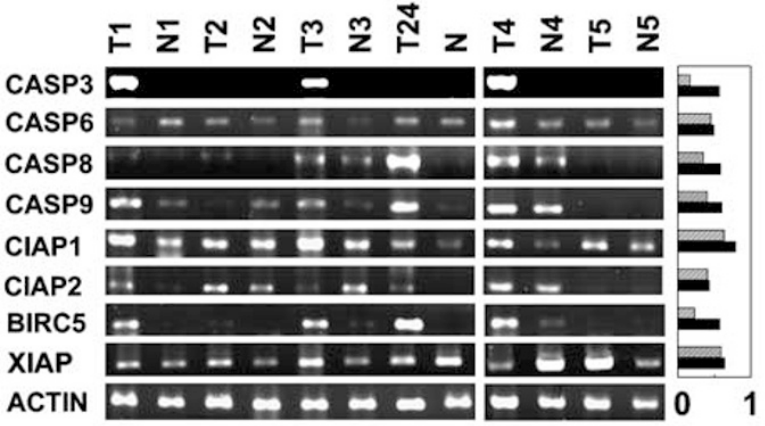

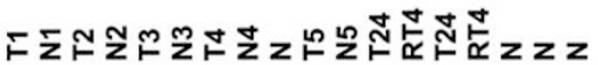

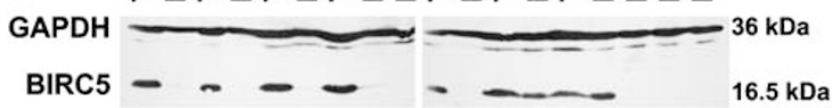

Figure 2 Upper panel. Caspase and IAP mRNA in urothelial carcinoma tissues (T1-T5), cell line (T24), and normal urothelium (N1-N5, and N) assessed by RT-PCR analysis. T1-T5 and N1-N5 were paired tumor and adjacent normal urothelium samples. $\mathrm{N}$ was an additional normal urothelium sample. The bar chart on the right represented comparison of average relative mRNA level (value range $0-1$ ) of each target gene in the tumor (black bars) and normal urothelium (hatched bars) samples. The average relative mRNA level was calculated from mRNA level of each gene relative to $\beta$-actin in each sample semiquantitatively by using ImageQuant (Molecular Dynamics). Lower panel. Survivin/BIRC5 protein in urothelial carcinoma tissues (T1-T5), cell lines (T24 and RT4), and in paired adjacent (N1-N5) and several additional normal urothelium samples $(\mathrm{N})$ assessed by Western blot analysis. GAPDH served as internal control of protein loading. BIRC5 was present in the tumor tissue or cell lines but virtually absent in normal urothelium.

a
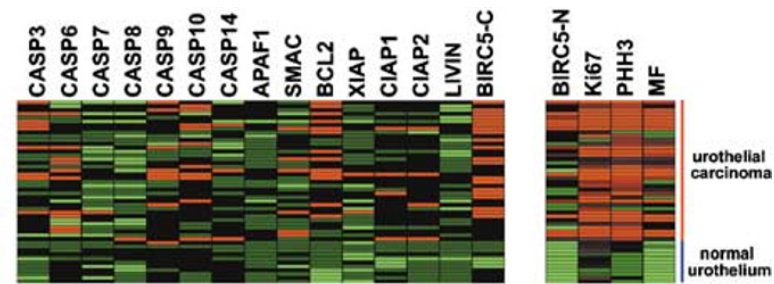

b

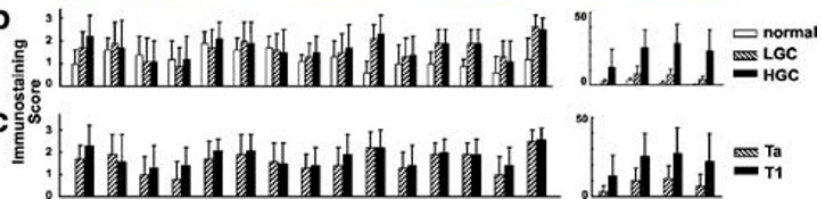

Figure 3 (a) Color scale map representation of immunostaining score of $37 \mathrm{Ta} / \mathrm{T} 1$ urothelial carcinomas and 11 normal urothelium samples. Cytoplasmic immunostaining (15 markers, left panel) is represented by green (score 0), deep green (score 1), black (score 2), and red (score 3). BIRC5-N (\%), Ki67 (\%), PHH3 and mitotic indexes (counts/10HPF) are scaled from green $(0 \%$ or $0 / 10 \mathrm{HPF}$ ) to red ( $>12 \%$ or $12 / 10 \mathrm{HPF}$ ). Each row represents data of one case. (b) Column chart comparison of each marker (mean + s.d.) in normal urothelium, LGC, and HGC. Differences between normal urothelium and carcinoma are statistically significant for CASP3, BCL2, CIAP1, CIAP2, BIRC5-C, BIRC5-N, and proliferation markers (all $P<0.01$, Mann-Whitney $U$-test). Differences between LGC and HGC are statistically significant only for BIRC5$\mathrm{N}$ and proliferation markers $(P<0.01$, Mann-Whitney $U$-test). (c) Column chart comparison of each marker (mean + s.d.) in Ta and T1 tumors. Only differences for BIRC5-N and proliferation markers are statistically significant $(P<0.01$, Mann-Whitney $U$-test).
BIRC5-N was not correlated with BIRC5-C. Figure 4 further highlighted the range of values as well as the significant difference of BIRC5-N and Ki67 labeling indexes between LGC and HGC, or between Ta and T1 tumors.

Noninvasive (Ta) low-grade urothelial carcinomas is considered 'genetically stable' and fundamentally different from 'genetically unstable' tumors, to which noninvasive (Ta) high-grade carcinomas and T1 tumors (regardless of grade) belong. ${ }^{3}$ Comparison was therefore also made between these two groups (group A: Ta LGC tumors; group B: either T1 or HGC). Group B ('genetically unstable' tumors) displayed significantly higher BIRC5-N labeling index $(P<0.01)$ than group A patients (Table 1$)$, but BIRC5-C immunostaining score was similar in these two groups.

\section{BIRC5-N, but not BIRC5-C, is of Prognostic Significance}

We further analyzed the prognostic value of BIRC5N, BIRC5-C, Ki67, tumor grade, stage, grouping based on 'genetic stability', and patient age, in this cohort of 101 pathologically confirmed Ta/T1

Table 1 Relationship between BIRC5-C immunoscore or BIRC5-N nuclear labeling index with clinicopathological variables

\begin{tabular}{|c|c|c|c|c|c|}
\hline & $\mathrm{n}^{*}$ & $\begin{array}{c}\text { BIRC5-C } \\
\text { (mean } \\
\text { score } \pm \text { s.d.) }\end{array}$ & P-value & $\begin{array}{c}\text { BIRC5-N } \\
(\%, \text { mean } \pm \text { s.d. })\end{array}$ & $\mathrm{P}$-value \\
\hline \multicolumn{6}{|l|}{ Gender } \\
\hline M & 81 & $2.0 \pm 0.8$ & & $6.6 \pm 7.8$ & \\
\hline $\mathrm{F}$ & 20 & $2.0 \pm 0.8$ & 0.7297 & $4.5 \pm 5.0$ & 0.2418 \\
\hline \multicolumn{6}{|l|}{ Age } \\
\hline$<67$ & 47 & $2.2 \pm 0.8$ & & $4.1 \pm 4.8$ & \\
\hline$\geq 67$ & 54 & $2.0 \pm 0.8$ & 0.2253 & $7.9 \pm 8.7$ & 0.0079 \\
\hline \multicolumn{6}{|l|}{ Grade } \\
\hline LGC & 59 & $2.2 \pm 0.7$ & & $2.7 \pm 2.8$ & \\
\hline HGC & 42 & $2.0 \pm 0.8$ & 0.2265 & $10.9 \pm 8.9$ & $<0.000$ \\
\hline \multicolumn{6}{|l|}{ Stage } \\
\hline $\mathrm{Ta}$ & 54 & $2.2 \pm 0.7$ & & $2.9 \pm 3.4$ & \\
\hline $\mathrm{T} 1$ & 47 & $2.0 \pm 0.9$ & 0.1180 & $9.9 \pm 8.8$ & $<\mathbf{0 . 0 0 0 1}$ \\
\hline \multicolumn{6}{|l|}{ Group } \\
\hline A & 45 & $2.2 \pm 0.7$ & & $2.3 \pm 2.2$ & \\
\hline B & 56 & $2.0 \pm 0.9$ & 0.2068 & $9.2 \pm 8.5$ & $<\mathbf{0 . 0 0 0 1}$ \\
\hline \multicolumn{6}{|l|}{ Ki67 } \\
\hline$<20 \%$ & 76 & $2.1 \pm 0.8$ & & $3.5 \pm 3.3$ & \\
\hline$\geq 20 \%$ & 25 & $2.0 \pm 0.8$ & 0.7216 & $14.2 \pm 10.1$ & $<\mathbf{0 . 0 0 0 1}$ \\
\hline \multicolumn{6}{|l|}{ BIRC5-C } \\
\hline$<2$ & 26 & $1.0 \pm 0.0$ & & $6.9 \pm 6.2$ & \\
\hline$\geq 2$ & 75 & $2.5 \pm 0.5$ & $<0.0001$ & $5.8 \pm 7.7$ & 0.5095 \\
\hline
\end{tabular}

${ }^{*} n$ : number of cases. s.d.: standard deviation. BIRC5-C and BIRC5-N denote cytoplasmic immune staining score and nuclear labeling index of BIRC5, respectively. Group A: only low-grade cases of Ta. Group B: cases of either HGC or T1. $P$-values $<0.05$ (by $t$-test) are highlighted in bold. 

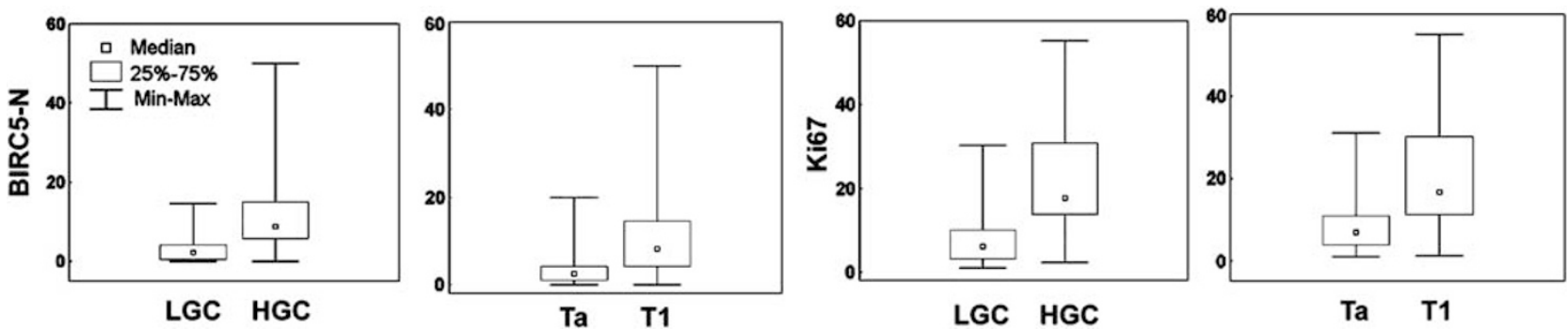

Figure 4 Comparison of BIRC5-N or Ki67 nuclear labeling index in $101 \mathrm{Ta} / \mathrm{T} 1$ tumors. For both markers, differences between LGC and HGC, or between Ta and T1, are statistically significant $(P<0.01)$.

patients of urinary bladder urothelial carcinoma, who received weekly intravesical instillation of BCG for 6 consecutive weeks, starting 2 weeks after complete resection of tumor by TURBT, without further adjuvant therapies. The median follow-up time for all cases was 54.0 months (10 and 90\% percentiles: 20.0 and 68.6 months, respectively) and that for censored cases was 60.2 months (10 and 90\% percentiles: 23.1 and 70.8 months, respectively). In this cohort, 19 patients died of recurrence or metastases, another six patients had pathologically confirmed recurrence but were either alive or dead of non-cancer-related causes. Kaplan-Meier method and log rank test were used for univariate analysis of disease-specific survival and progression-free survival, and Cox proportional hazard regression for multivariate analysis.

Univariate analyses showed that BIRC5-N $(\geq 8.0 \%$ vs <8.0), Ki67 labeling index (Ki67 $\geq 20 \%$ vs $<20 \%$ ), tumor grade (HGC vs LGC), stage (T1 vs Ta), and grouping based on 'genetic stability' were of prognostic significance with respect to both diseasespecific survival and progression-free survival (Figure 5, and Tables 2 and 3).

However, in multivariate analysis incorporating these parameters, only BIRC5-N retained independent prognostic power at a cutoff value of $8 \%$ (Tables 2 and 3), with approximately 4.4- to 6.6-fold increase of risk for progression or disease-specific death, respectively.

\section{Discussion}

Most earlier studies addressing apoptosis regulator expression in urothelial carcinomas of urinary bladder focused on BCL2 family, but its relationship with progression or patient outcome has not been settled. ${ }^{5,7-9,12,13,18,20}$ More recently, the potential role of two IAP family members in bladder cancer was studied by a few groups..$^{2,24,26,27}$ Gazzaniga et al, ${ }^{24}$ using RT-PCR, observed $30 \%$ positivity for BIRC5 and $23 \%$ for Livin in $\mathrm{Ta} / \mathrm{T} 1$ carcinomas. Weikert et $a l^{27}$ reported detection of BIRC5 mRNA in bladder cancer $(100 \%)$ but not in normal urothelium. $\mathrm{Ku}$ et $a 2^{26}$ reported high BIRC5 expression (defined as $>20 \%$ of tumor cells) in $58.0 \% \mathrm{Ta} / \mathrm{T} 1$ tumors. Bilim et $a 1^{22}$ observed weak XIAP expression in normal urothelium and usually stronger expression in $73.2 \%$ of urothelial carcinomas. Detection of BIRC5 in urine as an attractive, highly sensitive and specific marker of bladder cancer, has also been reported..$^{27,37,38}$

The expression status of other IAP family members and the caspases in Ta or T1 urothelial tumors has not been explored previously. The present study is the first to systematically evaluate expression of caspases, APAF1, SMAC, and the IAPs in Ta or T1 urothelial carcinomas. We demonstrated that urothelial carcinomas had significantly higher levels of some IAPs (BIRC5, CIAP1, and CIAP2) than normal urothelium. Interestingly, CASP3 was also increased in urothelial carcinomas. Overexpression of CASP3 has also been reported in hepatocellular carcinomas. ${ }^{39}$ Coexistence of high levels of CASP3 or CASP8 and BIRC5 or XIAP has been described in other tumor cell lines and tissues. ${ }^{40}$ Thus, our data showed IAPs and CASP3, being functionally adversarial molecules, could be coexpressed at higher levels in urothelial carcinoma than in normal urothelium as well.

However, with the exception of BIRC5-N, difference in expression of most apoptosis regulators (including BIRC5-C and other IAPs) between LGC and HGC, or between Ta and T1, or between 'genetically stable' and 'genetically unstable' urinary bladder urothelial carcinomas was not significant. This implicated that disturbed expression of most apoptosis regulators (including most IAPs, except BIRC5-N) in urinary bladder urothelial carcinomas as compared to normal urothelium probably represented common early events in urothelial carcinogenesis.

Relationship of proliferation activity with expression of most caspases and IAPs in urinary bladder urothelial carcinoma has not been previously explored. Our data showed that in Ta/T1 urothelial carcinoma proliferation activity was closely associated with BIRC5-N but not BIRC5-C or other apoptosis regulators. Also noticeable was that BIRC5 nuclear labeling index was not associated with other apoptosis regulators, including its own cytoplasmic staining (Table 1). It has been shown that, in addition to apoptosis regulation, BIRC5 was 
Disease-specific survival
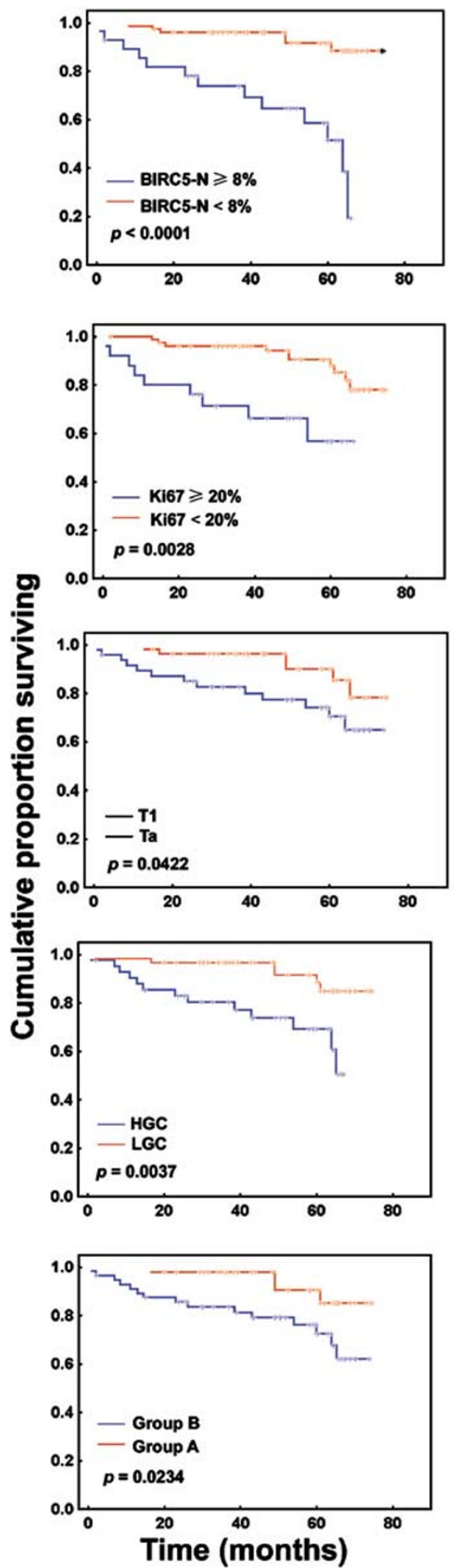

Progression-free survival
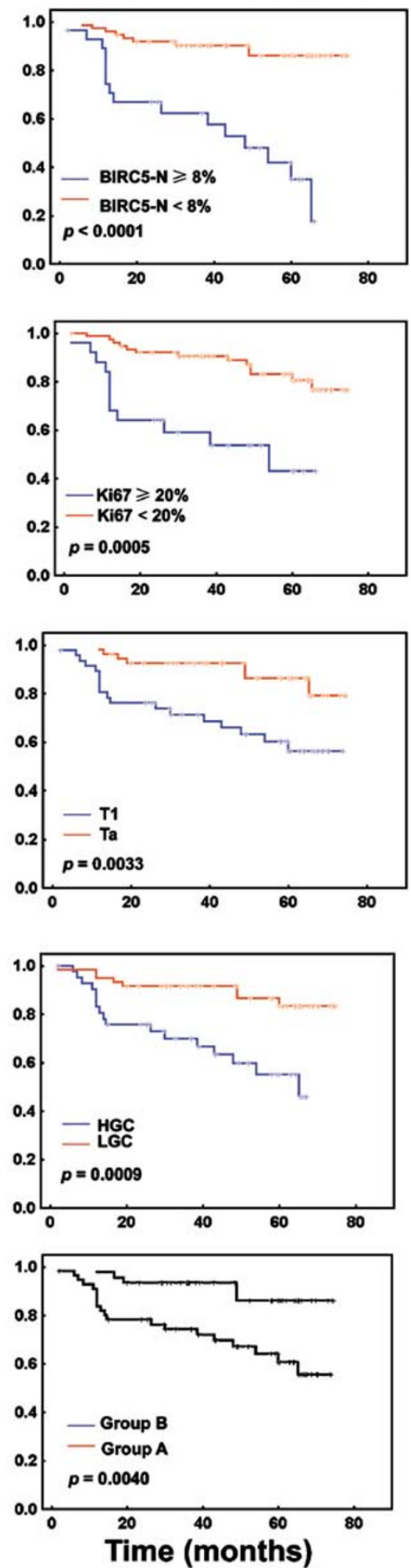

Figure 5 Survival analysis. Kaplan-Meier comparisons of disease-specific (left panels) or progression-free (right panels) survival for BIRC5-N, Ki67, stage, grade, and grouping based on 'genetically unstable' (group B, including cases of either HGC or T1) or 'genetically stable' (group A, including only low-grade cases of Ta). Log rank test $P$-values are listed for each parameter.

involved in control of mitosis and more generally in cell cycle progression. ${ }^{31,41,42}$ The existence of differential nuclear and cytoplasmic pools of BIRC5 and their functional diversification might be related to differential subcellular localization of BIRC5 splice variants. ${ }^{31,41,42}$ Importantly, it has also been noted 
Table 2 Disease-specific survival analysis

\begin{tabular}{|c|c|c|c|c|c|c|}
\hline & \multirow[t]{2}{*}{$\mathrm{n}^{*}$} & \multirow[t]{2}{*}{$\mathrm{P}($ Log rank) } & \multirow[t]{2}{*}{$\mathrm{P}$ (Cox regression) } & \multirow[t]{2}{*}{$R R$} & \multicolumn{2}{|c|}{$95 \%$ confidence interval } \\
\hline & & & & & Lower & Upper \\
\hline \multicolumn{7}{|l|}{ Age } \\
\hline$<67$ & 47 & & & & & \\
\hline$\geq 67$ & 54 & 0.1496 & 0.8368 & 1.1168 & 0.3904 & 3.1949 \\
\hline \multicolumn{7}{|l|}{ Grade } \\
\hline LGC & 59 & & & & & \\
\hline HGC & 42 & 0.0036 & 0.6193 & 1.5244 & 0.2900 & 8.0405 \\
\hline \multicolumn{7}{|l|}{ Stage } \\
\hline Ta & 54 & & & & & \\
\hline $\mathrm{T} 1$ & 47 & 0.0422 & 0.4941 & 1.7932 & 0.3363 & 9.5623 \\
\hline \multicolumn{7}{|l|}{ Group } \\
\hline A & 45 & & & & & \\
\hline B & 56 & 0.0234 & 0.6993 & 0.6335 & 0.0625 & 6.4216 \\
\hline \multicolumn{7}{|l|}{ Ki67 } \\
\hline$<20 \%$ & 76 & & & & & \\
\hline$\geq 20 \%$ & 25 & 0.0028 & 0.8307 & 1.1381 & 0.3477 & 3.7253 \\
\hline \multicolumn{7}{|l|}{ BIRC5-C } \\
\hline$<2$ & 26 & & & & & \\
\hline$\geq 2$ & 75 & 0.7587 & 0.1468 & 2.2858 & 0.7483 & 6.9826 \\
\hline \multicolumn{7}{|l|}{$B I R C 5-N$} \\
\hline$<8.0 \%$ & 73 & & & & & \\
\hline$\geq 8.0 \%$ & 28 & $<\mathbf{0 . 0 0 0 1}$ & 0.0080 & 6.6082 & 1.6375 & 26.6685 \\
\hline
\end{tabular}

that BIRC5 nuclear staining was mostly observed in a fraction of Ki67 positive cells, further supporting the idea that BIRC5 nuclear positivity was mainly related to cell proliferation. ${ }^{43}$ Thus, the close association with proliferative activity as well as tumor grade and stage in urothelial carcinomas indicated that BIRC5-N was probably more an indicator of proliferative advantage than antiapoptotic activity. Urothelial carcinoma progression, therefore, was probably more closely related to acquisition of additional proliferative advantages than to deregulated apoptosis. ${ }^{6}$

Only a few reports on association of IAPs with clinicopathological variables of urinary bladder urothelial carcinoma are available. One study noticed correlation of BIRC5 mRNA with grade but not stage in Ta/T1 tumors, and no correlation of Livin with either grade or stage. ${ }^{24}$ Another reported correlation of BIRC5 mRNA expression with tumor grade and stage (pTa-T3). ${ }^{27}$ Yet another found no correlation of BIRC5 immunostaining with grade or stage in $\mathrm{Ta} / \mathrm{T} 1$ carcinomas. $^{26}$ Reports regarding prognostic value of IAPs in urothelial carcinoma were scarce, ${ }^{22-24,26,27}$ and that of caspases were lacking. XIAP was not observed to correlate with prognosis of urothelial carcinomas. ${ }^{22}$ BIRC5 mRNA or protein expression was reported to be of prog- nostic significance by a few groups, ${ }^{23,26,27}$ but not by others. ${ }^{24}$ However, these studies did not specifically address the phenomenon and significance of differential nuclear and cytoplasmic expression of BIRC5 and its biologic prognostic significance in urothelial carcinomas. Only one study ${ }^{44}$ of urinary bladder urothelial carcinomas specified differential cytoplasmic and nuclear BIRC5 immunostaining, although the latter was not found to be statistically associated with prognosis in their series of 45 cases.

Different prognostic significance of BIRC5 nuclear labeling in several tumor types has been reported in recent years, ${ }^{4-50}$ as summarized in reference $\mathrm{Li}$ et al. ${ }^{51}$ For example, correlation of BIRC5 nuclear (but not cytoplasmic) staining with poor prognosis has been reported in ependymoma, esophageal squamous cell carcinoma, hepatocellular carcinoma, mantel cell lymphoma, and non-small cell lung cancer. ${ }^{45-48}$ Paradoxically, BIRC5 nuclear staining was reported to be associated with better prognosis in gastric cancer and breast cancer. ${ }^{49,50}$ It has been proposed that variations in immunostaining and interpretation might be among the contributing factors to these conflicting results. ${ }^{51}$

The distinct nuclear and cytoplasmic staining in our study is unequivocal, and we provided detailed quantitative data on the nuclear staining than 
Table 3 Progression-free survival analysis

\begin{tabular}{|c|c|c|c|c|c|c|}
\hline & \multirow[t]{2}{*}{$n^{*}$} & \multirow[t]{2}{*}{$\mathrm{P}($ Log rank) } & \multirow[t]{2}{*}{$\mathrm{P}$ (Cox regression) } & \multirow[t]{2}{*}{$R R$} & \multicolumn{2}{|c|}{$95 \%$ confidence interval } \\
\hline & & & & & Lower & Upper \\
\hline \multicolumn{7}{|l|}{ Age } \\
\hline$<67$ & 47 & & & & & \\
\hline$\geq 67$ & 54 & 0.2218 & 0.9540 & 0.9742 & 0.3996 & 2.3744 \\
\hline \multicolumn{7}{|l|}{ Grade } \\
\hline LGC & 59 & & & & & \\
\hline HGC & 42 & 0.0009 & 0.7127 & 1.3199 & 0.3013 & 5.7819 \\
\hline \multicolumn{7}{|l|}{ Stage } \\
\hline Ta & 54 & & & & & \\
\hline $\mathrm{T} 1$ & 47 & 0.0033 & 0.3982 & 1.9674 & 0.4093 & 9.4556 \\
\hline \multicolumn{7}{|l|}{ Group } \\
\hline A & 45 & & & & & \\
\hline B & 56 & 0.0040 & 0.8129 & 0.7781 & 0.0974 & 6.2148 \\
\hline \multicolumn{7}{|l|}{ Ki67 } \\
\hline$<20 \%$ & 76 & & & & & \\
\hline$\geq 20 \%$ & 25 & 0.0005 & 0.6920 & 1.2357 & 0.4338 & 3.5198 \\
\hline \multicolumn{7}{|l|}{ BIRC5-C } \\
\hline$<2$ & 26 & & & & & \\
\hline$\geq 2$ & 75 & 0.5356 & 0.4869 & 1.3756 & 0.5599 & 3.3796 \\
\hline \multicolumn{7}{|l|}{$B I R C 5-N$} \\
\hline$<8.0 \%$ & 73 & & & & & \\
\hline$\geq 8.0 \%$ & 28 & $<\mathbf{0 . 0 0 0 1}$ & 0.0151 & 4.4137 & 1.3334 & 14.6101 \\
\hline
\end{tabular}

previous reports. Our data clearly showed that BIRC5 nuclear labeling index, but not cytoplasmic staining, was significantly associated with tumor grade, stage, and poorer prognosis for Ta/T1 urothelial carcinomas. Although Ki67 was found to be more powerful a prognosticator than BIRC5-N in intracranial ependymoma, ${ }^{43}$ our multivariate analysis indicated that BIRC5-N was a superior prognostic marker to Ki67, tumor grade, and stage for Ta/T1 urinary bladder urothelial carcinoma. These more classical parameters did not show strong independent prognostic value (even when BIRC5-N was not included) in the multivariate analysis. For Ki67, this probably reflected the greater variability of Ki67 in urothelial carcinomas, which reduced its power to predict tumor behavior. The narrower range and closer correlation of BIRC5-N staining with aggressive behavior made it an apparently better prognosticator for Ta/T1 urinary bladder urothelial carcinoma, as can be appreciated by comparing the survival curves (Figure 5). BIRC5-N labeling index might find its way into clinical use as a very useful biologic marker than existing ones.

As most Ta/T1 urinary bladder urothelial carcinomas showed high cytoplasmic BIRC5 levels, as demonstrated by our data, analysis of total BIRC5 protein probably would be less powerful as a biomarker in Ta/T1 urothelial carcinomas. Indeed, in our initial analysis of BIRC5 immunostaining when a total immunoscore combining cytoplasmic and nuclear staining was used, no association with tumor grade, stage, or prognosis could be appreciated based on such integrated immunoscores of BIRC5. Although Western blot analysis of BIRC5 protein is of value in confirming the presence of the target protein, it would not have distinguished nuclear and cytoplasmic expression unless subcellular fractionation was performed. RT-PCR analysis of mRNA also would be less useful in this respect, since it is well known that mRNA levels may not always correlate with protein levels. Thus, BIRC5 immunostaining is of particular use in this scenario as it differentiates the two pools of BIRC5, and that evaluation of BIRC5 cytoplasmic and nuclear immunostaining must be separately scored to maximize its use as a biomarker.

\section{Acknowledgements}

We dedicate this paper to professor Guanghua Yang, mentor of us for decades, who has just passed away. We thank Dr P Zhang and other colleagues in the Department of Urology, Dr Y Wang, Ms Y Deng and Ms D Xiao for technical assistance, Dr B Yang, Mr K Yang, Ms B Liu, and Ms X Wu for retrieving archival material. We are most grateful to colleagues who reviewed earlier drafts of the manuscript for their 
insights and comments, particularly with regard to the potential biologic significance of BIRC5 nuclear labeling. Supported by grants to QZ from the Natural Science Foundation of China (NSFC, 30125023, 30570692, 30221001) and the Ministry of Science and Technology (2002CCA01400) of China.

\section{References}

1 Jemal A, Murray T, Ward E, et al. Cancer statistics. CA Cancer J Clin 2005;55:10-30.

2 Stein JP, Grossfeld GD, Ginsberg DA, et al. Prognostic markers in bladder cancer: a contemporary review of the literature. J Urol 1998;160:645-659.

3 Eble JN, Sauter G, Epstein JI, et al. (eds). World Health Organization classification of tumours. Pathology and genetics of the urinary system and male genital organs. IARC Press: Lyon, France, 2004, pp 89-123.

4 Bush C, Price P, Norton J, et al. Proliferation in human bladder carcinoma measured by Ki-67 antibody labeling: its potential clinical importance. Br J Cancer 1991;64:357-360.

5 Glick SH, Howell LP, White RW. Relationship of p53 and bcl-2 to prognosis in muscle-invasive transitional cell carcinoma of the bladder. J Urol 1996;155: 1754-1757.

6 King ED, Matteson J, Jacobs SC, et al. Incidence of apoptosis, cell proliferation and bcl-2 expression in transitional cell carcinoma of the bladder: association with tumor progression. J Urol 1996;155:316-320.

7 Shiina $\mathrm{H}$, Igawa $\mathrm{M}$, Urakami S, et al. Immunohistochemical analysis of bcl-2 expression in transitional cell carcinoma of the bladder. J Clin Pathol 1996;49: 395-399.

8 Gazzaniga P, Gradilone A, Vercillo R. Bcl-2/bax mRNA expression ratio as prognostic factor in low-grade urinary bladder cancer. Int J Cancer 1996;69:100-104.

9 Lipponen PK, Aaltomaa S, Eskelinen M. Expression of the apoptosis suppressing bcl-2 protein in transitional cell bladder tumours. Histopathology 1996;28: 135-140.

10 Liukkonen T, Rajala P, Raitanen M, et al. Prognostic value of MIB-1 score, p53, EGFr, mitotic index and papillary status in primary superficial (Stage pTa/T1) bladder cancer: a prospective comparative study. Eur Urol 1999;36:393-400.

11 Tsuji M, Kojima K, Murakami Y, et al. Prognostic value of Ki-67 antigen and p53 protein in urinary bladder cancer: immunohistochemical analysis of radical cystectomy specimens. Br J Urol 1997;79:367-372.

12 Nakopoulou L, Vourlakou C, Zervas A, et al. The prevalence of bcl-2, p53, and Ki-67 immunoreactivity in transitional cell bladder carcinomas and their clinicopathological correlates. Hum Pathol 1998;29: 146-154.

13 Tzai TS, Chow NH, Lin JS, et al. The expression of p53 and bcl-2 in superficial bladder transitional cell carcinoma and its role in the outcome of postoperative intravesical chemotherapy. Anticancer Res 1998;18: $4717-4721$

14 Zlotta AR, Noel JC, Fayt I, et al. Correlation and prognostic significance of p53, p21WAF1/CIP1 and Ki67 expression in patients with superficial bladder tumors treated with bacillus Calmette-Guerin intravesical therapy. J Urol 1999;161:792-798.
15 Pfister C, Lacombe L, Vezina MC, et al. Prognostic value of the proliferative index determined by Ki-67 immunostaining in superficial bladder tumors. Hum Pathol 1999;30:1350-1355.

$16 \mathrm{Wu} \mathrm{TT}$, Chen JH, Lee YH, et al. The role of bcl-2, p53, and ki-67 index in predicting tumor recurrence for low grade superficial transitional cell bladder carcinoma. J Urol 2000;163:758-760.

17 Gontero P, Casetta G, Zitella A, et al. Evaluation of P53 protein overexpression, Ki67 proliferative activity and mitotic index as markers of tumour recurrence in superficial transitional cell carcinoma of the bladder. Eur Urol 2000;38:287-296.

18 Korkolopoulou P, Lazaris ACh, Konstantinidou AE, et al. Differential expression of bcl-2 family proteins in bladder carcinomas. Relationship with apoptotic rate and survival. Eur Urol 2002;41:274-283.

19 Rodel C, Grabenbauer GG, Rodel F, et al. Apoptosis, p53, bcl-2, and Ki-67 in invasive bladder carcinoma: possible predictors for response to radiochemotherapy and successful bladder preservation. Int J Radiat Oncol Biol Phys 2000;46:1213-1221.

20 Ong F, Moonen LM, Gallee MP, et al. Prognostic factors in transitional cell cancer of the bladder: an emerging role for Bcl-2 and p53. Radiother Oncol 2001;61: 169-175.

21 Rodriguez-Alonso A, Pita-Fernandez S, GonzalezCarrero J, et al. p53 and ki67 expression as prognostic factors for cancer-related survival in stage T1 transitional cell bladder carcinoma. Eur Urol 2002;41: 182-188.

22 Bilim V, Kasahara T, Hara N, et al. Role of XIAP in the malignant phenotype of transitional cell cancer (TCC) and therapeutic activity of XIAP antisense oligonucleotides against multidrug-resistant TCC in vitro. Int J Cancer 2003;103:29-37.

23 Schultz IJ, Kiemeney LA, Witjes JA, et al. Survivin mRNA expression is elevated in malignant urothelial cell carcinomas and predicts time to recurrence. Anticancer Res 2003;23:3327-3331.

24 Gazzaniga P, Gradilone A, Giuliani L, et al. Expression and prognostic significance of LIVIN, SURVIVIN and other apoptosis-related genes in the progression of superficial bladder cancer. Ann Oncol 2003;14: 85-90.

25 Lopez-Beltran A, Luque RJ, Alvarez-Kindelan J, et al. Prognostic factors in stage T1 grade 3 bladder cancer survival: the role of G1-S modulators (p53, p21Waf1, p27kip1, Cyclin D1, and Cyclin D3) and proliferation index (ki67-MIB1). Eur Urol 2004;45: 606-612.

$26 \mathrm{Ku} \mathrm{JH}$, Kwak C, Lee HS, et al. Expression of survivin, a novel inhibitor of apoptosis, in superficial transitional cell carcinoma of the bladder. J Urol 2004;171: 631-635.

27 Weikert S, Christoph F, Schrader M, et al. Quantitative analysis of survivin mRNA expression in urine and tumor tissue of bladder cancer patients and its potential relevance for disease detection and prognosis. Int J Cancer 2005;116:100-104.

28 Salvesen GS, Abrams JM. Caspase activation-stepping on the gas or releasing the brakes? Lessons from humans and flies. Oncogene 2004;23:2774-2784.

29 Shi Y. Caspase activation, inhibition, and reactivation: a mechanistic view. Protein Sci 2004;13:1979-1987. 
30 Riedl SJ, Shi Y. Molecular mechanisms of caspase regulation during apoptosis. Nat Rev Mol Cell Biol 2004;5:897-907.

31 Schimmer AD. Inhibitor of apoptosis proteins: translating basic knowledge into clinical practice. Cancer Res 2004;64:7183-7190.

32 Salvesen GS, Duckett CS. IAP proteins: blocking the road to death's door. Nat Rev Mol Cell Biol 2002;3: 401-410.

33 Wang $\mathrm{X}$. The expanding role of mitochondria in apoptosis. Genes Dev 2001;15:2922-2933.

34 Fuessel S, Kueppers B, Ning S, et al. Systematic in vitro evaluation of survivin directed antisense oligodeoxynucleotides in bladder cancer cells. J Urol 2004; 171:2471-2476.

35 Miller RT, Kubier P. Blocking of endogenous avidinbinding activity in immunohistochemistry: The use of skim milk as an economical and effective substitute for commercial biotin solutions. Appl Immunohistochem Mol Morphol 1999;7:63-65.

36 Nielsen TO, Hsu FD, O’Connell JX, et al. Tissue microarray validation of epidermal growth factor receptor and SALL2 in synovial sarcoma with comparison to tumors of similar histology. Am J Pathol 2003; 163:1449-1456.

37 Smith SD, Wheeler MA, Plescia J, et al. Urine detection of survivin and diagnosis of bladder cancer. JAMA 2001;285:324-328.

38 Shariat SF, Casella R, Khoddami SM, et al. Urine detection of survivin is a sensitive marker for the noninvasive diagnosis of bladder cancer. J Urol 2004; 171:626-630.

39 Persad R, Liu C, Wu TT, et al. Overexpression of caspase-3 in hepatocellular carcinomas. Mod Pathol 2004;17:861-867.

40 Yang L, Cao Z, Yan H, et al. Coexistence of high levels of apoptotic signaling and inhibitor of apoptosis proteins in human tumor cells: implication for cancer specific therapy. Cancer Res 2003;63:6815-6824.
41 Li F, Ambrosini G, Chu EY, et al. Control of apoptosis and mitotic spindle checkpoint by survivin. Nature 1998;396:580-584.

42 Altieri DC. Molecular circuits of apoptosis regulation and cell division control: the survivin paradigm. J Cell Biochem 2004;92:656-663.

43 Preusser M, Wolfsberger S, Czech T, et al. Survivin expression in intracranial ependymomas and its correlation with tumor cell proliferation and patient outcome. Am J Clin Pathol 2005;124:543-549.

44 Lehner R, Lucia MS, Jarboe EA, et al. Immunohistochemical localization of the IAP protein survivin in bladder mucosa and transitional cell carcinoma. Appl Immunohistochem Mol Morphol 2002;10:134-138.

45 Grabowski P, Kuhnel T, Muhr-Wilkenshoff $\mathrm{F}$, et al. Prognostic value of nuclear survivin expression in oesophageal squamous cell carcinoma. Br J Cancer 2003;88:115-119.

$46 \mathrm{Lu} \mathrm{B}$, Gonzalez A, Massion PP, et al. Nuclear survivin as a biomarker for non-small-cell lung cancer. Br J Cancer 2004;91:537-540.

47 Martinez A, Bellosillo B, Bosch F, et al. Nuclear survivin expression in mantle cell lymphoma is associated with cell proliferation and survival. Am J Pathol 2004;164:501-510.

48 Fields AC, Cotsonis G, Sexton D, et al. Survivin expression in hepatocellular carcinoma: correlation with proliferation, prognostic parameters, and outcome. Mod Pathol 2004;17:1378-1385.

49 Okada E, Murai Y, Matsui K, et al. Survivin expression in tumor cell nuclei is predictive of a favorable prognosis in gastric cancer patients. Cancer Lett 2001; 163:109-116.

50 Kennedy SM, O’Driscoll L, Purcell R, et al. Prognostic importance of survivin in breast cancer. Br J Cancer 2003;88:1077-1183.

51 Li F, Yang J, Ramnath N, et al. Nuclear or cytoplasmic expression of survivin: what is the significance? Int J Cancer 2005;114:509-512. 